other laboratories which may be in a position to do so to undertake comparable measurements.

On the subjects of electric and photometric units, the Conference had little to do except to take note of, and confirm, the decision already taken by the International Committee, in pursuance of the instructions of the previous Conference, that the new units should come into force as from January 1, 1948. It may be worth while to recall that the relationship of the old to the new (absolute) electric units is 1 mean international ohm, $\Omega_{M}=1 \cdot 00049$ absolute ohm; 1 mean international volt $V_{M}=$ 1.00034 absolute volt; and that the new unit of candle-power, which in future is to be designated 'candela', is defined as "such that the brightness of an integral (black body) radiator, at the temperature of solidification of platinum, equals 60 candela per square centimetre".

With regard to thermometry, the Conference accepted a revised text of the International remperature Scale, recommended by the International Committee on the advice of its consultative committee. This is a very detailed statement. It may be useful to record that the primary fixed points of the scale are :

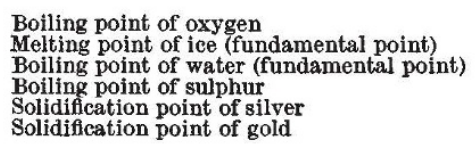

Temperature

$-182 \cdot 970$

100

100
$444 \cdot 600$

$444 \cdot 600$
$960 \cdot 8$

$1063 \cdot 0$

Temperatures in the revised scale are to be quoted as ${ }^{\circ} \mathrm{C}$. (Int. 1948). It should also be mentioned that, after discussion, it was unanimously agreed to describe temperatures in this scale as degrees "Celsius" instead of Centigrade or Centesimal. Objections were raised to both the latter on different grounds.

Turning to a more fundamental question, it was agreed that the thermodynamic scale should, in principle, be based on a single fixed point, namely, the triple point of pure water, which should have the value +0.0100 degree, together with the absolute zero, the value of which should be fixed at a later date, when more precise measurements are available.

It was also agreed that the unit of heat should be the 'Joule', and it was remitted to the International Committee to establish a table relating the value of various 'calories' to the Joule, in accordance with the best available experimental data.

The Conference also referred to the Committee the task of instituting an inquiry among all the contracting States as to the opinions held with regard to the possible formulation of a common practical system of units of measurement suitable for adoption by all countries using the metric system, and of making recommendations.

Following an initiative of the International Union of Physics, the Conference adopted a table of nomenclature and symbols relating to a selected group of quantities which were held to come within its domain. It also approved a proposed recommendation that, so far as Europe is concerned, words ending in ". . . illion" should be accepted as referring to successive powers of $10^{6}$.

Approval was given to a proposed agreement for mutual co-operation between the International Committee and the United Nations Educational, Scientific and Cultural Organisation on questions of common interest.
The Conference next addressed itself to the regulation of the scale of subscriptions for the various countries adhering to the Convention. Proposals for further extensions of the activities of the Bureau were put forward by the Russian delegation and referred to the Committee for study. A suggestion was made by the Swiss delegate that the Governments of the contracting States might be invited to supplement their statutory contributions by voluntary donations to facilitate such additional undertakings.

Finally, the Conference proceeded to the re-election of the International Committee. Many members of the Committee elected in 1933 have since died. In addition to seven former members who retain their seats, the following were elected, or re-elected after retirement by rule: L. de Broglie (French), G. Cassinis (Italian), E. C. Crittenden (American), M. Dehalu (Belgian), W. J. de Haas (Dutch), T. Isnardi (Argentine), H. Nagaoka (Japanese), Z. Rauszer (Polish), M. Siegbahn (Swedish).

The Conference passed in an atmosphere of friendly co-operation, and it will be seen that it accomplished successfully a large programme of work covering a very wide field.

\section{EVOLUTION OF COLOUR VISION}

$\Delta$ DIscussion if evolution of colour vision A took place at he general meeting of the Linnæan Society on Februgry 24. Dr. R. J. Pumphrey, while disclaiming pxpeft knowledge, said that it seemed fitting that foolog/sy/should have been invited to open the doctsion ather than a specialist in human colour yision: ho Ayer knowledgeable, since a zoological (twining enfrces a historical outlook, which is essential for the ultimate understanding of the evarion of function. The evidence available from animals other than mammals, though extremely relevant, is still scanty; inferences from structure and physiology cannot be relied on. Colour vision can only be ascribed to an animal when the intact animal, either in the field or in a controlled experimental situation, has been shown to exercise a choice depending on colour discrimination. Such a test is always a matter of extreme technical difficulty, and is not applicable to any animal without restriction but only to those whose habits and motivation make them suitable subjects. In spite of these limitations, colour vision has been demonstrated beyond reasonable doubt in a number of insects, notably aculeate Hymenoptera, Diptera and Lepidoptera, and a number of vertebrates, notably teleost fish, reptiles, birds and primates.

This demonstration leads immediately to two obvious questions. To what extent is the fundamental mechanism of colour vision similar in these animals? And to what extent is it utilized in a similar way? To the first question, the best available answer comes from measurements of the least perceptible difference in wave-length $(\Delta \lambda)$ of pure spectral colours as a function of wave-length. This function is of identical form for man (Wright), pigeon (Hamilton and Coleman) and minnow (Wolff). The maximum and minimum values of $\Delta \lambda$ occur at the same values of wave-length $\lambda$. For the bee the curve has the same general character but is displaced, one minimum value of $\Delta \lambda$ being in the ultra-violet near $3800 \mathrm{~A}$. $(\mathrm{Kühn})$. It is reasonable to conclude that in all these cases colour vision depends on the existence of three 
or more types of photoreceptor with different absorption spectra. None of these curves has the least resemblance to the discrimination curve of a human dichromat, although it has been suggested that dichromatism is an essential stage in the evolution of full colour vision.

It does not follow, however, that colour vision has the same character in all these animals. Kühn's work appears to show that the effect known as simultaneous colour contrast, which is of trivial importance in human vision, is dominant for the bee. If this be the case, no object of small angular subtense in the bee's visual field will be intrinsically coloured; its apparent hue will depend primarily on the background against which it is seen.

The human eye unaided cannot distinguish spectral green from a suitable mixture of spectral blue and yellow, but a match so made will no longer be a match when seen through a coloured filter. A proportion of the cones of a bird's retina contains red and yellow oil droplets each of which is a colour filter for its own cone. Hence it is obviously physically possible for a bird to distinguish impure colours which are indistinguishable to man without ex. traneous aids. These examples indicate that in spite of a fundamentally similar mechanism of colour vision in different animals, the quality and extent of the information about the environment which their eyes collect need not be the same or even similar.

The fact that colour vision appears to be demonstrable in just those animals whose habits lend it a particular importance suggests that the fundamental mechanism is an essential attribute of any highly organised eye, and one which may or may not be exercised in the direction of colour vision according to need. Hartline's and Granit's demonstration of receptors of different spectral sensitivities in the retinæ of a number of animals which are certainly colour-blind supports this conclusion.

Dr. E. N. Willmer said that, in spite of many attempts, colour vision had not been demonstrated in any mammal outside the primates. The primate stock was in all probability nocturnal in habits, with a retina dominated by rods, a rod being defined as a visual receptor cell containing rhodopsin and being maximally sensitive at $500 \mu \mu$. A cone contains a pigment other than rhodopsin and is maximally sensitive at $570 \mu \mu$. Histologically, although they are typically very different, they may in certain species and situations be difficult to distinguish. Moreover, rods themselves may differ widely in their power to accumulate rhodopsin, and Granit has demonstrated the existence of distinguishable groups of high- and low-threshold rods in cats and guinea pigs. It is probable that, in view of their evolutionary history, the mechanism of colour vision in mammals may be quite different from that of birds, which is probably wholly dependent on cones. Dr. Willmer said he would submit evidence that in primates colour vision depends on rods and cones ; neither alone can produce the sensation of colour.

In the colour vision of primates, the most important part of the retina is the central part of the macula lutea, which is called the fovea, and subtends an angle of about $2^{\circ}$. Within the fovea is the central 'rod-free' area subtending less than $1^{\circ}$, with greatly diminished power of dark-adaptation and containing no lowthreshold rods. The normal fovea in man is trichromatic and must contain at least three types of receptor elements with separate pathways to the colour-analysing centre of the brain. The rod-free area, however, behaves as a dichromatic system. Within it all spectral colours can be matched by mixtures of red and violet.

Subjects who confuse red and green (both protanopes and deuteranopes) are completely colour. blind in the rod-free area. Any spectral hue can here be matched to any other by altering its intensity only. They differ, however, in the spectral sensitivity of this area, protanopes being relatively insensitive to red and deuteranopes falling into two classes, normal (type I) and relatively insensitive to blue (type II), respectively. It is inferred that the sensitivity curves of the protanope and the deuteranope (type II) represent respectively the spectral sensitivity of the two elements normally present in the rod-free area. For the deuteranope (type II) the inference is strongly supported by the way in which the thresholds for red and violet light respectively are modified by previous exposure to high intensities of red or violet. The deuteranope of type I has normal sensitivity and adaptation, indicating that both 'red' and 'green' receptors are present in the central rodfree area; but he is colour-blind because their output is not recognized as distinct by the central anklyser. The deuteranope (type II) is colour-blind in the central fovea because only the 'red' receptors are present. Further, the sensitivity curve of the 'red' receptors is not so unlike the absorption spectrum of iodopsin as to exclude the idea that the 'red' receptors are, in fact, cones in which iodopsin is the sensitive pigment. In spite of certain complications in the adaptation results, it is probable that the protanope is colour-blind in the rod-free area because only 'green' receptors are present.

Histologically, two trends are distinguishable in the evolution of the primate retina : the specialization of the rod + mop-bipolar system for dim-light vision and of the cone + midget bipolar in the centre of the retina for good acuity at higher illumination. These lead ultimately to the existence of three pathways in the eye as a whole and two in the central fovea. The pathways are formed by the mopbipolars relaying from low-threshold rods (the 'blue' pathway), the flat bipolars relaying from high. threshold rods and cones (the 'green' pathway), and the midget bipolars relaying from cones only (the 'red' pathway).

Both histological evidence of the evolution of the retina in primates and the evidence from the exam. ination of colour-defect in man, Dr. Willmer said, are consistent with the existence of three types of receptor only, namely, cones, high-threshold rods and low-threshold rods, the two first only being present in the 'rod-free' area.

Admiral T. P. H. Beamish stressed the continuing importance for the Services of research on colour vision, relating that his own yeoman of signals had been found to be colour-blind after thirteen years of service. Dr. L. C. Thomson pointed out that Dr. Willmer was relying heavily on the fact that protanopes are totally colour-blind in the rod-free area. In Dr. Thomson's experience, this was not altogether true and a time factor was involved. The subject is conscious of a colour sensation initially, which changes or vanishes on continued fixation.

Dr. H. J. A. Dartnall raised the question whether the absorption spectrum of iodopsin is sufficiently close to the sensitivity curve of the deuteranope, type II, to justify the assumption that human cones depend on iodopsin. 
Prof. H. Hartridge said that he could confirm the old observation of Holmgren that a bright yellow point centrally fixated could be seen as red, white, green or yellow and a blue point as green, white, blue or violet. How was it possible to reconcile this observation with the existence of only two receptor types in the central fovea?

Dr. M. H. L. Pirenne suggested that more use might be made of the opto-motor reaction in investi. gating the colour-vision of animals by a method similar to that used by Hecht in determining acuity.

Dr. Willmer, in reply to Dr. Thomson and Prof. Hartridge, said that it was not really relevant to argue about whether or not a colour sensation was aroused in the subject. The crucial question was whether the sensation, whatever its subjective nature, could be matched tri-, di- or mono-chromatically.

Dr. Pumphrey, in reply to Dr. Pirenne, said that the difficulties of the technique suggested, if not insuperable, were so formidable that suspicion might attach to the results. The method had, in fact, been tried by Birukow on the frog with results in conflict with those of other workers.

R. J. P.

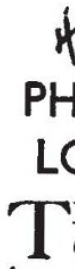
the

\section{PHYSICAL AND PSYCHO-PHYSIO- LOGICAL ASPFCTS OF COLOUR}

THE British Psylo logical Society and the Colour Group of the Hysical Society held a joint meeting, their first, in the Hastings Hall, Tavistock House, London, on Feb? ruary $1 \%$, when a number of problems about colouf were fisteussed. Mr. J. G. Holmes, chairmap of th Colour Group, was in the chair. Threg man topics were introduced by members of a pand onsisting of Dr. L. C. Thomson (Vision Research Unit, Medical Research Council), Mr. R. C. Oldfield (Institute of Experimental Psychology, Oxford), Dr. R. W. Pickford (Department of Psychology, Glasgow), Dr. W. D. Wright (Department of Optics, Imperial College of Science and Technology, London) and Mr. W. A. Allen (Building Research Station, Department of Scientific and Industrial Research). General discussion of each subject followed the contributions of the panel.

Dr. Thomson spoke first, on "The Measurement and Meaning of the Luminosity Curve". He emphasized the difficulties of determining accurately the forms of this curve, and outlined the two principal methods, namely, $(a)$ obtaining brightness matches for a series of monochromatic stimuli against a standard monochromatic stimulus; (b) determining the absolute intensity-threshold for a series of wavelengths. By these methods the photopic and scotopic curves are obtained, and their form and relative position in the spectrum suggest a number of problems, of which Dr. Thomson detailed two. The first of these is that of the shift in the position of the luminosity curve as conditions are changed from scotopic to photopic, and in this connexion he made reference to Dartnall's suggestion that such a shift might be produced by selective absorption by increasing concentrations of the breakdown product of rhodopsin-indicator yellow-under increasing light intensities. Such a hypothesis would, he remarked, predict a limited shift of about the correct amount. The second problem about the luminosity ocigrte raised by Dr. Thomson was that of the secondary maxima, or 'humps' found on the photopic curve. $\mathrm{He}$ pointed out that if these are connected with independent colour mechanisms, doubts must arise regarding that part of the Young-Helmholtz trichromatic theory which postulates that a sensation of white is the product of the activity of the three colour receptors alone, and of no other mechanism.

Mr. Oldfield referred briefly to some of the problems, physical and psychological, which arise concerning the position of the luminosity curve in the electro. magnetic spectrum, and the biological utility of colour vision. He cited Wall's view that, since the vertebrate eye evolved in an aqueous environment, the absorption spectrum of water must be one of the factors determining the position and extent of the sensitivity curve of the eye. He mentioned other physical factors which impose similar limitations. On the question of the biological utility of colour vision, he referred to the difficulties of determ. ining accurately its incidence among animal species, and remarked upon its somewhat odd occurrence. He suggested that its chief utility lies in the enormous variety of discriminable patterns of visual stimulation that it affords, compared to the resources offered by mere intensity discrimination. He asked whether the actual incidence of colour mechanisms might not be bound up with the food habits of the species con. cerned.

Dr. Pickford spoke next on "The Inadequacy of Existing Tests of Colour Vision". He remarked first that most tests are vitiated by the fact that they were devised with a theory of colour defect in mind. Indeed, he said, most would be quite useless if the phenomena of colour-blindness did not show marked discontinuities. The tests are satisfactory only in so far as they are responsive to distinctions between the colour-blind and the normal. They have little capacity to separate out the anomalies. He then briefly criticized a number of tests in common use. Holmgren's wool-test had been effectively disposed of by Edridge-Green, who showed that it failed in many cases to detect defects of occupational significance. Tests of the Stilling and Ishi-Hara type serve to reveal the chief discontinuous classes of colour defect, but scarcely do much to distinguish the protanope from the deuteranope. Edridge-Green's bead test depends on naming, and no test should depend on this alone; further, its yellow-blue section is ineffective, and the test generally is too easily passed by defectives. Lantern tests such as those of Edridge-Green and Martin depend on naming, itself a complex function. They also suffer from extreme intricacy of procedure, if the instructions are fully complied with. Spectrometer tests, again, rely on the expression of discrimination in terms of names, and suffer from the disadvantage that they require an experienced worker to administer them. They are also unable to deal with the red-green anomalies. The anomaloscope, finally, is excellent for the detection and measurement of red-green anomalies, but is unsuitable for use on other types of defect.

Dr. Wright maintained that the available tests are not entirely unsatisfactory, provided they are used for purposes to which they are suited. In occupational testing it is important in the first place to pick out the grosser defects, and to select those subjects who require further testing of a more special character. The Ishi-Hara test and the anomaloseripistingether will achieve this. The more special tests should be devised so as to provide as close an imitation of the occupational working conditions as possible. Dr. Wright emphasized that, as an oceu- 